\title{
CONTRAST VARIATION WITH RESONANT SOFT X-RAY SCATTERING FOR SOFT MATERIALS
}

\author{
Cheng Wang
}

Advanced Light Source, Lawrence Berkeley National Laboratory; CWang2@lbl.gov

An improved understanding of fundamental chemistry, morphology, and dynamics in polymers and soft materials requires advanced characterization techniques that amenable to in situ and in operando studies. Soft X-ray methods are especially useful in their ability to non-destructively provide material or chemical moiety specific information. Recent development of resonant soft x-ray scattering (RSoXS) at the Advanced Light Source (ALS) has enabled its applications to many critical research areas of materials research. Combining conventional x-ray scattering with soft x-ray absorption spectroscopy, RSoXS is a unique chemical sensitive structure probe that provides a novel route to unambiguously decipher the complex morphologies of mesoscale materials. Tuning $x$-ray photon energies to match the absorption spectrum of the different chemical components, the scattering contributions from the different components can be selectively enhanced, enabling a glimpse into these complex morphologies with unprecedented details. Applications of RSoXS have been extended to the areas of structured polymer assemblies, organic electronics, functional nano-composites, liquid crystals, as well as bio/bio-hybrid materials. Recent development of customized instrumentation, multimodal characterization methods, as well as complementary theory and modeling for the extraction of the chemical distribution and spatial arrangement at multiple length scales in the application of soft materials will be discussed.

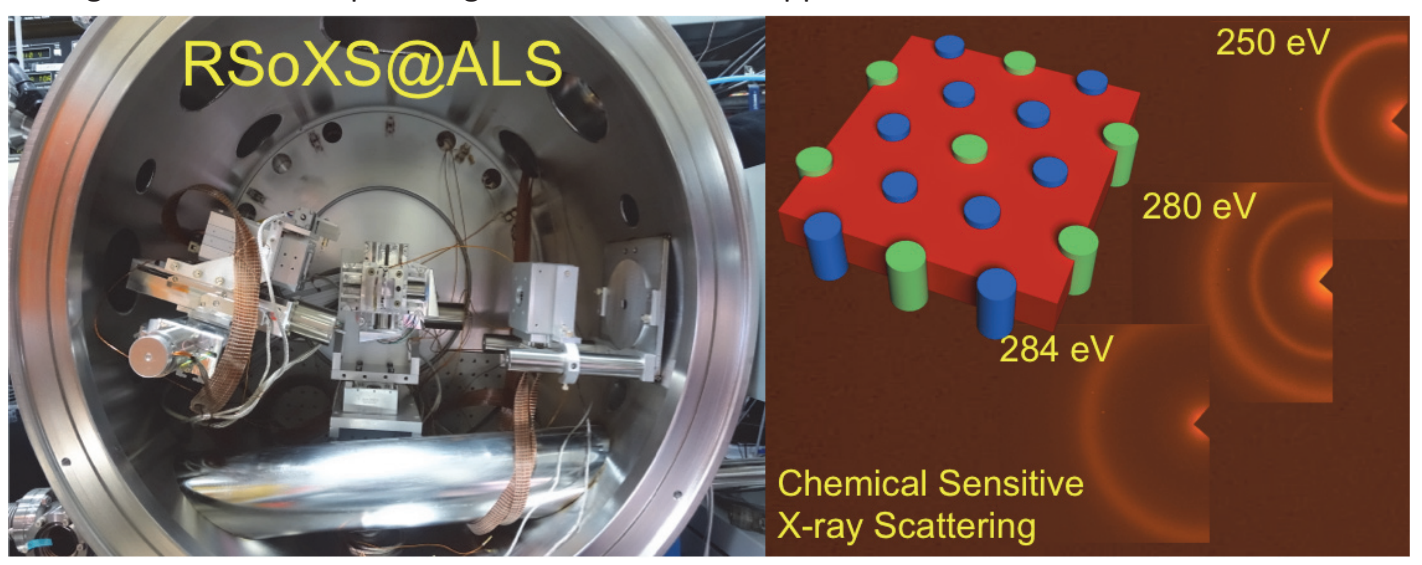

